\title{
Hydrogen Storage Enhancement Attained by Fixation of Ti on MWNTs
}

\author{
J. J. Pérez-Bueno, ${ }^{1}$ M. L. Mendoza López, ${ }^{2}$ K. M. Brieño Enriquez, ${ }^{1}$ J. Ledesma García, ${ }^{3}$ L. A. \\ Godínez Mora-Tovar, ${ }^{1}$ and C. Angeles Chavez ${ }^{4}$ \\ ${ }^{1}$ Centro de Investigación y Desarrollo Tecnológico en Electroquímica, S.C. Subdirección de Investigación, Parque Tecnológico Querétaro- \\ Sanfandila, 76703 Pedro Escobedo, QRO, Mexico \\ ${ }_{2}^{2}$ Departamento de Metalmecánica, Instituto Tecnológico de Querétaro, Avenida Tecnológico s/n Esq. M. Escobedo, Col. Centro, 76000 \\ Querétaro, QRO, Mexico \\ ${ }^{3}$ División de Investigación y Posgrado, Facultad de Ingeniería, Universidad Autónoma de Querétaro (UAQ), Centro Universitario, \\ 76010 Querétaro, QRO, Mexico \\ ${ }^{4}$ Programa de Ingeniería Molecular, Instituto Mexicano del Petróleo, Eje Central Lázaro Cárdenas 152, A. P. 14-805, 07730 México City, \\ DF, Mexico
}

Correspondence should be addressed to J. J. Pérez-Bueno, jperez@cideteq.mx

Received 23 March 2012; Revised 8 June 2012; Accepted 17 June 2012

Academic Editor: Jasmina Grbovic Novakovic

Copyright ( 2012 J. J. Pérez-Bueno et al. This is an open access article distributed under the Creative Commons Attribution License, which permits unrestricted use, distribution, and reproduction in any medium, provided the original work is properly cited.

\begin{abstract}
Nowadays, hydrogen has a preponderant position among the potentially sustainable energy sources. Due to its power density, its storage is of main concern when considering a broad use in practical applications. Carbon nanotubes constitute promising candidates for the design and construction of hydrogen storage devices. This work explores the use of some procedures involving electrochemistry, aimed to bond atomic Ti on the outer surface of MWNTs. Each titanium atom has the potential of hosting two hydrogen molecules and relinquishing them by heating. Nevertheless, nanotubes are difficult to handle due to electrostatic charge and agglomeration, and in this context, two routes were tested as procedures to spread and stick nanotubes on an electrode: (1) a functionalization capable of attaching gold was tested in two forms, as either using $4 \mathrm{~nm}$ particles or a flat gold electrode. The fixation of Au particles was confirmed by HRTEM. (2) A simpler route that consisted on drying a $\mathrm{CH}_{2} \mathrm{Cl}_{2} /$ nanotubes solution previously spread on a glassy carbon flat electrode. $\mathrm{CH}_{2} \mathrm{Cl}_{2}$ was selected as the medium and $\mathrm{TiCl}_{4}$ as the precursor for attaching atomic Ti to the nanotubes. The results revealed that hydrogen adsorption, estimated from voltamperometry, was five times higher on Ti-MWNTs than on bare nanotubes.
\end{abstract}

\section{Introduction}

It is widely accepted that hydrogen possesses the potential to become the renewable energy source of the future. Nevertheless, some challenges must be addressed in order to allow its broad use in everyday items, and probably the one that stands out as the most important corresponds to the safe storage issue. Since the discovery of carbon nanotubes, countless attempts have been made in order to use them as cheap, safe, and practical materials for the storage of hydrogen [1-3]. Their capacity for direct hydrogen storage was determined to be around $6.5 \mathrm{wt} \%$ [4-6]. However, in order to increase this capacity, different MWNTs surface modifying methods have been proposed. In the case of surface functionalization for improving hydrogen storage, the main objective is to promote the fixation of metallic particles capable of attaching hydrogen atoms, which can be released when desired. In this way, there are reports of different metallic [7-11], nonmetallic, or bimetallic [1214] nanoparticles attached to nanotubes (carbon [7-10, 12], gold $[11,15]$, and others $[16,17])$. Some reports are based on an increase in catalytic performance $[7,13,14]$ or in 


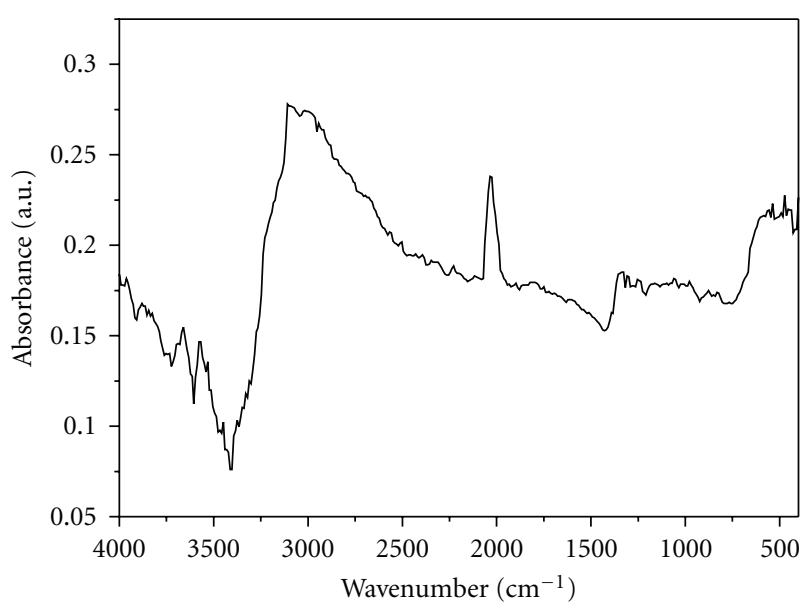

FIGURE 1: IR spectrum corresponding to functionalized nanotubes.

the sensitivity $[8-10,12]$. In this context, numerous groups have been studying electrochemical hydrogen storage [1824]. There are for instance reports on the catalytic effect of nickel nanoparticles located inside nanotubes for hydrogen dissociation [25-27].

The basis of the present study lies on previous works that report the feasibility of attaching two hydrogen atoms to an each Ti atom bonded to the outer surface of a MWNT [28-32]. In this way, each bonded titanium atom has the potential to host up to two hydrogen atoms or four hydrogen molecules and release them by heating. In this work, the functionalization of gold substrates was studied in order to find an alternative way to spread and stick nanotubes on an electrode surface. In addition, a simpler procedure based on drying a $\mathrm{CH}_{2} \mathrm{Cl}_{2} / \mathrm{MWNT}$ solution on the surface of a flat carbon electrode was studied.

\section{Experimental}

MWNTs are easier to obtain than single-walled carbon nanotubes (SWNTs) by different techniques and therefore the former were chosen for this work. The MWNTs were synthesized by pyrolyzing ferrocene/melamine mixtures at $1050^{\circ} \mathrm{C}$ in an Ar atmosphere. The carboxylic functionalization of nanotubes implies a series of steps that progressively modifies the surface changing their inert nature [14]. In this way, MWNTs underwent chemical treatment using a sulfuric acid/nitric acid mixture $(3: 1 \mathrm{v} / \mathrm{v})$ in an ultrasonic bath for two hours.

In a second step, the pretreated nanotubes were mixed with a cationic polyelectrolyte, polydiallyldimethylammonium (PDADMAC, mMw 100,000-200,000) dissolved in an aqueous $\mathrm{NaCl}$ solution (5\%) for $30 \mathrm{~min}$. This process at the same time prevents the aggregation of the MWNTs and allows joining the polyelectrolyte with the modified nanotubes by electrostatic interaction of the formed carbonyl groups and the PDADMAC. Finally, the nanotubes were mixed with either an aqueous colloid of $4-6 \mathrm{~nm}$ gold particles for $30 \mathrm{~min}$ or deposited on a flat gold electrode (Au coated glass).

An alternative simplified route that allowed electrochemical attachment of the $\mathrm{Ti}$ species was also explored. This consisted of drying a previously sonicated nanotube solution carefully spread on a flat glassy carbon electrode. Four liquid media were tested for these experiments: toluene $\left(\mathrm{C}_{6} \mathrm{H}_{5}-\mathrm{CH}_{3}, 99.8 \%\right)$, dichloromethane $\left(\mathrm{CH}_{2} \mathrm{Cl}_{2}, 99.9 \%\right)$, dichloroethane $\left(\mathrm{ClCH}_{2} \mathrm{CH}_{2} \mathrm{Cl}, 99 \%\right)$, and tetrahydrofuran (- $\mathrm{CH}_{2} \mathrm{CH}_{2}-\mathrm{O}-\mathrm{CH}_{2} \mathrm{CH}_{2}-$ ) 99.9\%), all from Sigma-Aldrich.

The MWNTs modified Au or glassy carbon electrodes were incorporated as working electrodes in a standard threeelectrode glass cell, where Ag and Pt wires were used as reference and counter electrodes, respectively. High purity $\mathrm{N}_{2}$ and $\mathrm{H}_{2}$ gases were used in all the experiments (both 99.999\% pure).

Electrochemical oxidation, by means of anodic polarization, provided functional groups susceptible to nucleophilic attack, which allowed interaction of the surface with a titanium precursor. This requires a high reactivity to effectively bond titanium atoms to nanotubes. The chosen titanium precursor was titanium(IV) chloride $\left(\mathrm{TiCl}_{4}\right.$, Sigma-Aldrich 99.995\%). In order to avoid titania formation, the procedure was conducted at $5^{\circ} \mathrm{C}$ in a glovebox with an Ar atmosphere.

Anodic polarization at different potentials between 0 and $+1500 \mathrm{mV}$ (versus $\mathrm{Ag}$ wire) for $5 \mathrm{~min}$ was therefore conducted in dry $\mathrm{CH}_{2} \mathrm{Cl}_{2}$. Then, $40 \mu \mathrm{L}$ of $\mathrm{TiCl}_{4}$ were injected directly in the electrochemical cell and mixed under constant stirring, in order to promote the immobilization of Ti atoms on the surface of the MWNTs. After $30 \mathrm{~s}$ of reaction, the electrode was withdrawn from the electrochemical cell and rinsed with sufficient $\mathrm{CH}_{2} \mathrm{Cl}_{2}$ to remove the $\mathrm{TiCl}_{4}$ excess. Later, the modified electrode was immersed in a $\mathrm{H}_{2}$-satured $\mathrm{CH}_{2} \mathrm{Cl}_{2}$ solution. Finally, the characterization was carried out by cyclic voltammetry, recorded from 1.5 to $-2 \mathrm{~V}$ at a scan rate of $0.05 \mathrm{Vs}^{-1}$ in a $\mathrm{CH}_{2} \mathrm{Cl}_{2} 10 \mathrm{mM}$ solution of Tetra $n$ butyl-ammonium hexafluoro-phosphate (TBAHFP) under $\mathrm{N}_{2}$-atmosphere. Glassy carbon electrodes without MWNTs or $\mathrm{TiCl}_{4}$ were used as control references.

The analysis of modified MWNTs was carried out using a Tecnai G2 F30 S-Twin HRTEM operating at $300 \mathrm{kV}$. The microscope is equipped with a Schottky-type field emission gun and an S-Twin objective lens (Cs, $1.2 \mathrm{~mm}$; Cc, $1.4 \mathrm{~mm}$; resolution, $0.20 \mathrm{~nm}$ ). Samples were pulverized and suspended in 2-propanol at room temperature and dispersed with ultrasonic agitation. Subsequently, aliquots of the solution were dropped on $3 \mathrm{~mm}$ diameter lacey carbon copper grids.

\section{Results and Discussion}

The chemical treatment described in the experimental section promotes the oxidation of the MWNTs surface forming carbonyl $\left(\mathrm{RR}^{\prime} \mathrm{C}=\mathrm{O}\right)$, carboxyl $(-\mathrm{C}(=\mathrm{O}) \mathrm{OH})$, and hydroxyl $(\mathrm{R}-\mathrm{O}-\mathrm{H})$ groups. As can be seen in Figure 1, the infrared spectrum shows the presence of characteristic peaks for carbonyl groups around $1600 \mathrm{~cm}^{-1}$. The band at $3300 \mathrm{~cm}^{-1}$ is related to carboxyl units while the bands at $1500 \mathrm{~cm}^{-1}$ and 


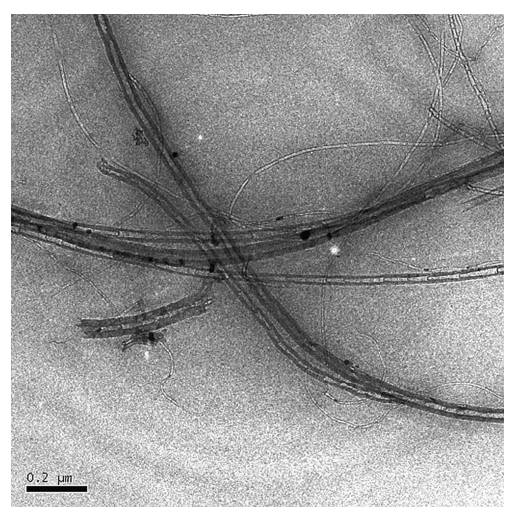

(a)

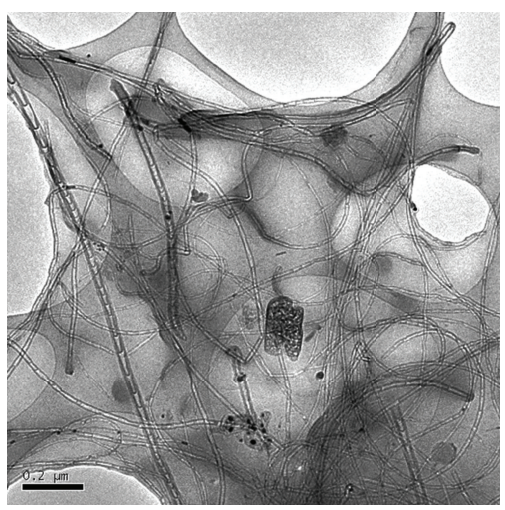

(b)

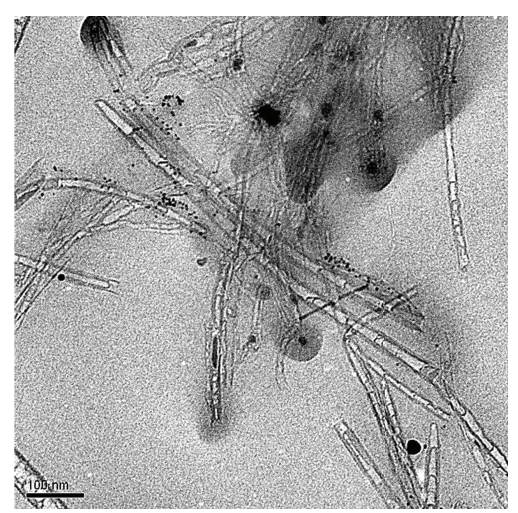

(c)

FIGURE 2: Multiwall carbon nanotubes: (a) modified with Au particles, (b, c) modified with Ti (not evident in the image) after sample preparation procedure for HRTEM.

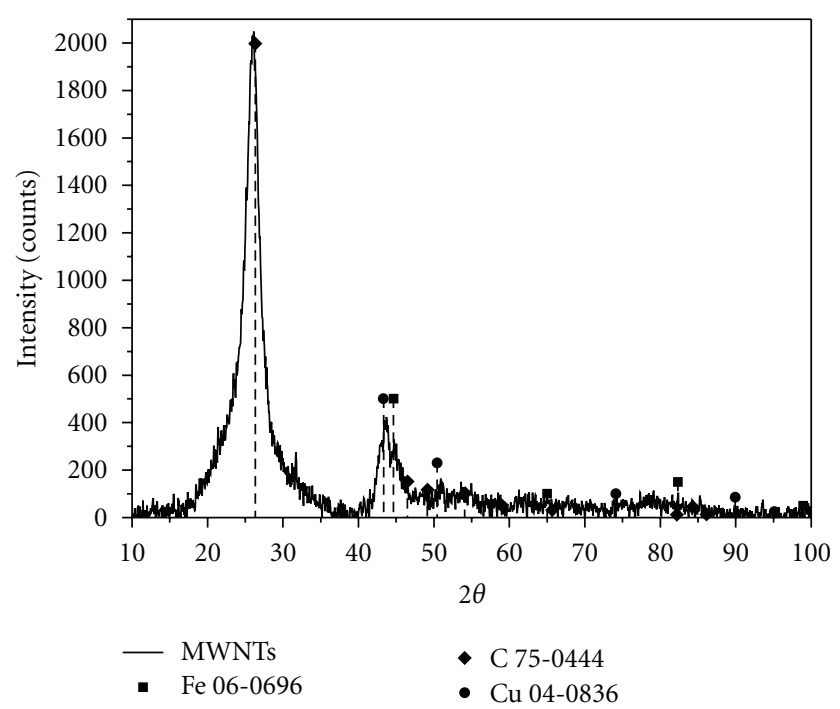

(a)

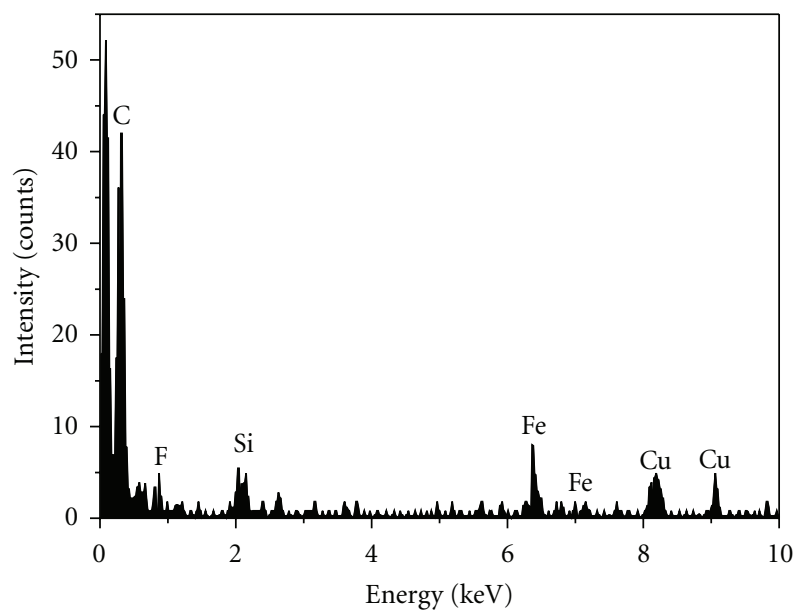

(b)

Figure 3: (a) Diffractogram of MWNTs indicating the corresponding JCPDS patterns, and (b) EDX corresponding to a point selected by HRTEM.
$1300 \mathrm{~cm}^{-1}$ correspond to $\mathrm{C}=\mathrm{C}$. The band at $1100 \mathrm{~cm}^{-1}$ is associated to the $\mathrm{C}-\mathrm{O}$ vibration.

Although the $\mathrm{Ti}$ modification of MWNTs was not evident in the HRTEM image, the close examination of the synthesized carbon nanotubes mainly shows multiwall type structures (Figure 2). Figure 3(a) shows an X-ray diffraction analysis of the bare MWNTs, which shows $\mathrm{C}$ and $\mathrm{Fe}$ corresponding to the nanotubes and the residual iron, respectively. The modified MWNTs, on the other hand, showed no differences in the X-ray diffraction analysis. From this observation, the presence of copper was not clear since its main peak position coincides with that of iron. The full widths at half maximum (FWHM) for the peaks were $2.312\left(\mathrm{C} ; 26.1 ; 2-\mathrm{Theta}^{\circ}\right), 1.54\left(\mathrm{Fe} ; 44.7 ; 2\right.$-Theta $\left.{ }^{\circ}\right)$, and 1.55 $\left(\mathrm{Cu} ; 43.6 ; 2-\mathrm{Theta}^{\circ}\right)$. The estimated composition (using the software TOPAS 4.2 ) was $67.16 \%$ graphite, $31.54 \%$ carbon, $1.0 \%$ iron, and $0.3 \%$ copper. The proportion of the MWNTs was around $70 \%$ with the remaining $30 \%$ of amorphous carbon, which coincides with that estimated for graphite and crystalline carbon, respectively. The corresponding crystallite sizes were $4.0 \mathrm{~nm}, 0.6 \mathrm{~nm}, 5.0 \mathrm{~nm}$, and $38.2 \mathrm{~nm}$, respectively. Similarly, in Figure 3(b), EDX analysis shows that C and Fe prevail but that another element appeared, copper.

As it was previously mentioned, the PDADMAC functionalized MWNTs were obtained. The fixation of nanotubes to Au was attained as described in the experimental section. Two cases using PDADMAC were tested, either attaching $4 \mathrm{~nm} \mathrm{Au}$ particles to the nanotubes, or by linking the nanotubes-polyelectrolyte complex to a gold electrode. The first case, already profusely reported in the literature, results in MWNTs decorated with Au nanoparticles (4 nm in diameter), which do not allow polarization to promote $\mathrm{Ti}$ attachment. The same procedure in a reversed condition allowed on the other hand, a proven way of attaching the MWNTs to a conducting Au electrode, where Ti implantation and polarization trough voltamperometry can be carried out. The test consisted of functionalizing the MWNTs with PDADMAC and attaching the resulting complex to a flat gold electrode in order to conduct a voltametry test. 
The resulting experiments established that the presence of the large chains of the polyelectrolyte probably screened the MWNTs charged groups, thus resulting in a hindering effect for the following step of titanium fixation. Therefore, the construction of a modified Au electrode with MWNTs anchored through PDADMAC functionalization, proved to be a nonconvenient route for Ti electrochemical attachment. Instead, another simplified route was chosen.

A simplified alternative procedure consisted of drying a sonicated MWNTs solution on a glassy carbon flat electrode as described in the experimental section. Once the carbon electrode surface was modified, anodic polarization for electrochemical oxidation was carried out. This step provided functional groups on the MWNTs susceptible to nucleophilic attack by the titanium precursor, $\mathrm{TiCl}_{4}$.

The titanium precursor requires high reactivity to effectively bond the titanium atoms to the nanotubes. At the same time, the synthetic protocol must avoid the formation of titania. In this context, titanium tetrachloride met these criteria and was chosen for this process. However, we required an inert medium, due to the precursor's reactivity with moisture and oxygen. As an alternative to handling this situation, we kept the medium at a low temperature (around $5^{\circ} \mathrm{C}$ ), which allowed working even in an air atmosphere.

On a regular basis, the experiments were conducted in an $\mathrm{Ar}$ chamber with four liquid media at a temperature around $5^{\circ} \mathrm{C}$ : toluene, dichloromethane, dichloroethane, and tetrahydrofuran. Dichloromethane was selected to be the best among them. $\mathrm{TiCl}_{4}$ showed precipitates with the other liquid media, titanium complex formations, or long time reactions probably occurred with the other solvents. In the case of dichloroethane, there was a sudden reaction with $\mathrm{TiCl}_{4}$ forming a white precipitate and the reaction kept going on for days. The THF-TiCl 4 reaction formed a yellowish gel. Toluene reacted with $\mathrm{TiCl}_{4}$ in 24 hours, forming first an orange solution and, subsequently, a precipitate.

Figure 4(a) shows the voltamperograms with the bare carbon electrode using $\mathrm{NaCl} 5 \% / \mathrm{wt}$ and with the polyelectrolyte (with waves at $0.74 \mathrm{~V}$ and $0.61 \mathrm{~V}$ ). Figure $4(\mathrm{~b})$ shows the voltamperogram (three cycles) of an electrode containing MWNTs, which showed a reduction characteristic wave located at $-0.23 \mathrm{~V}$.

The use of an ultramicroelectrode, with aperture diameter of $11 \mu \mathrm{m}$, was proposed for conducting the electrochemical study with the Ti modified MWNTs (Figure 5). The idea of using such a small electrode was to avoid the need to employ an electrolyte prevent impurities reduction, and/or incompatibilities with the Ti precursor. An ultramicroelectrode has a semispherical diffusion profile, which induces an improvement in the mass transport due to its small diameter. The MWNTs were placed on the electrode surface as described in the experimental section and typical voltamperometric responses are shown in Figure 5. However, the quantity of MWNTs in the ultra-microelectrode for each test was not reproducible and the associated voltamperogram currents were different in magnitude for each experiment. Also, the nanotubes surpassed the diffusion layer due to their length, which reduced the advantages of using an ultramicroelectrode for this study.

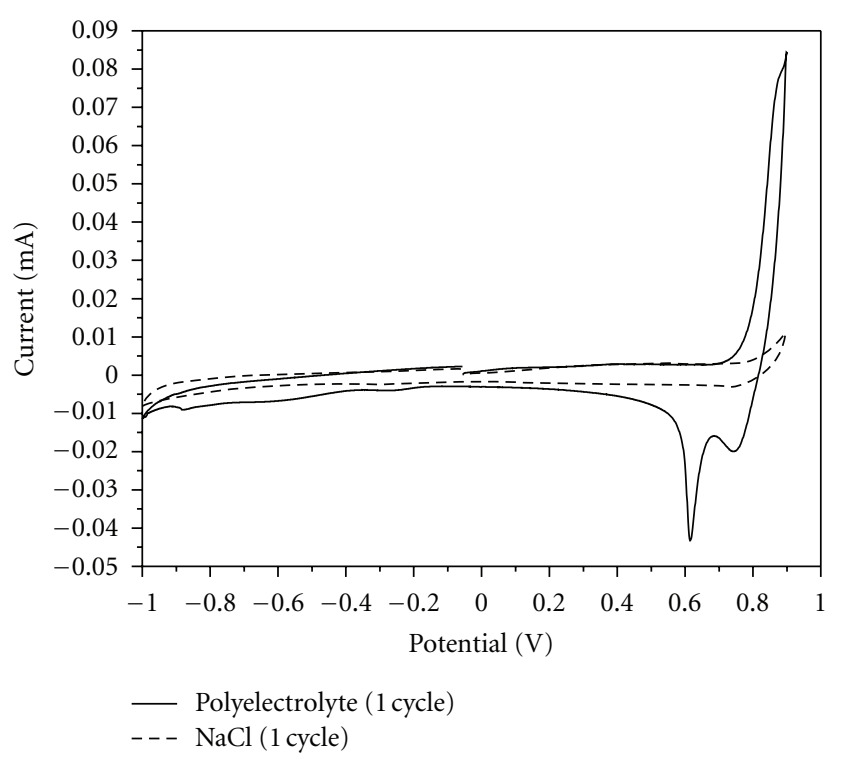

(a)

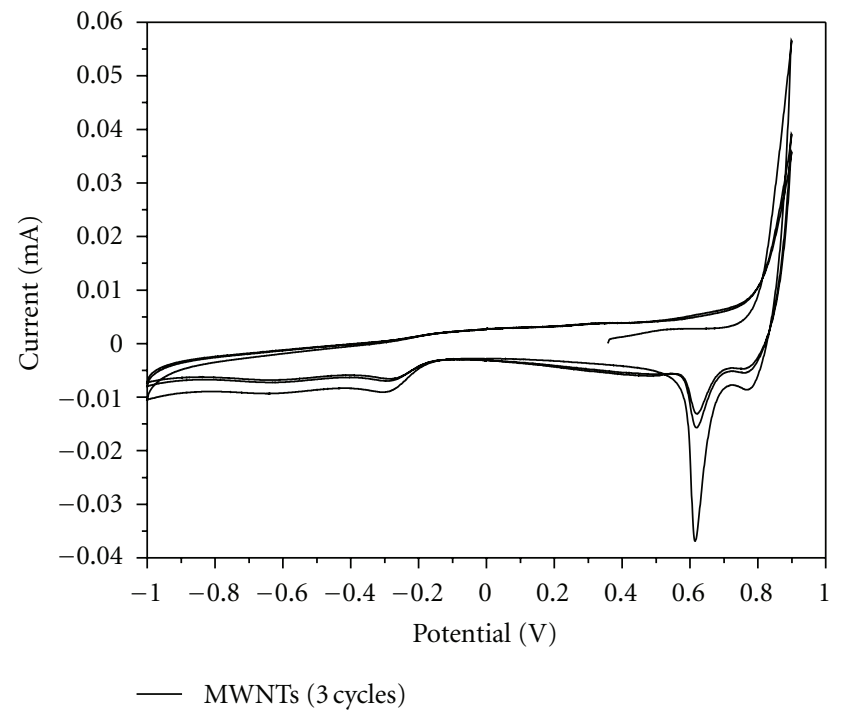

(b)

FigURE 4: Voltamperograms corresponding to (a) bare electrolyte and polyelectrolyte, as well as (b) functionalized nanotubes with the polyelectrolyte versus $\mathrm{Ag} / \mathrm{AgCl}$.

As can be seen in Figure 6, the resulting voltamperograms show the redox process associated with hydrogen adsorptiondesorption. It is important to note that a substantial increase in the current density is associated with the presence of Timodified MWNTs in the electrode surface. As previously mentioned, the carbon nanotubes have a reversible hydrogen storage capacity, but when Ti is incorporated in the MWNT surface, this capacity is considerably increased.

On the other hand, the electrochemical response of a Ti-modified MWNT electrode, previously immersed in $\mathrm{N}_{2}$-satured $\mathrm{CH}_{2} \mathrm{Cl}_{2}$ was also obtained (see Figure 6). As expected, this modified electrode does not present any redox reactions associated with the hydrogen adsorptiondesorption process. 


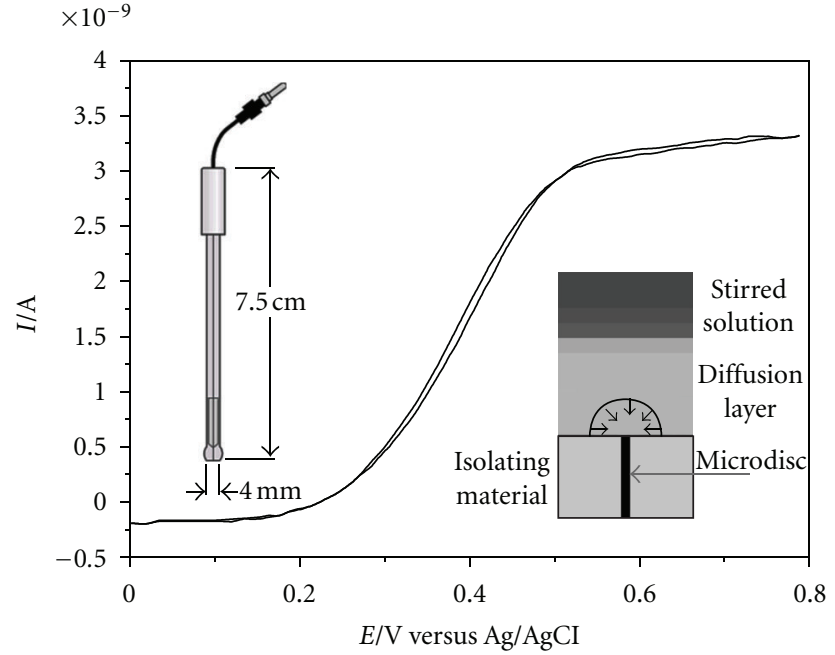

(a)

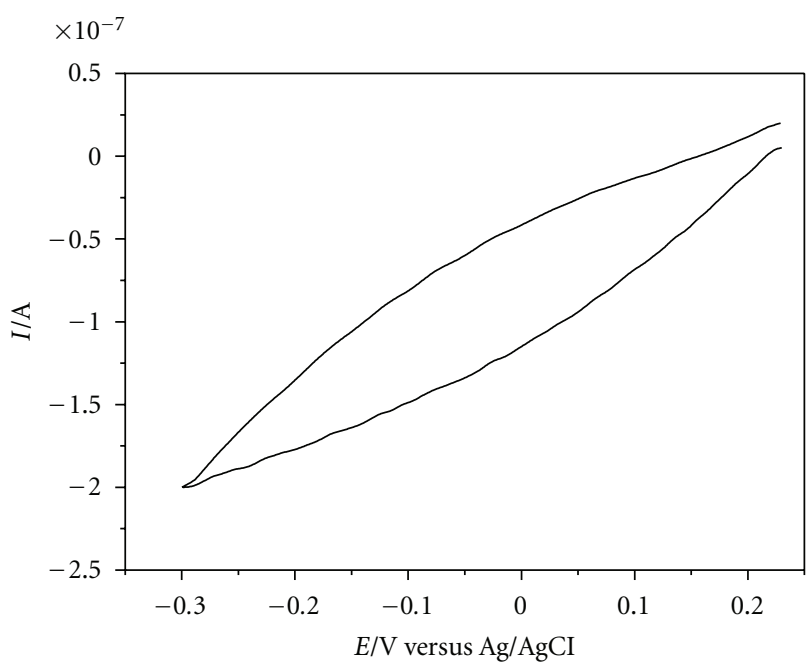

(b)

FIGURE 5: Ultramicroelectrode voltametry (a) without, and (b) with MWNTs. The inserted figures give a graphic representation of an ultramicroelectrode. Graphic representations of an ultramicroelectrode are shown.

Figure 7(a) shows the influence of anodic polarization on the modified MWNT electrode for Ti immobilization. The charge associated with hydrogen desorption in the voltamperograms was estimated by means of the integration of the area under the reduction peak located in the potential range of $0.1-1.8 \mathrm{~V}$ (Figure $7(\mathrm{~b})$ ) and adjusted by subtraction of the double-layer charging current. Each cyclic voltamperogram was obtained with Ti-modified MWNTs previously treated by application of different anodic potential values.

Therefore, anodic polarization pretreatment increased the capacity of Ti-modified MWTNs to attached $\mathrm{H}$ atoms on the electrode surfaces. This result could be directly related with the Ti coverage on the nanotube surfaces. Analysis of this data shows that when an anodic potential of $750 \mathrm{mV}$ was imposed on the MWNT surfaces, the anchorage of Ti is more favorable than the other potentials surveyed, and

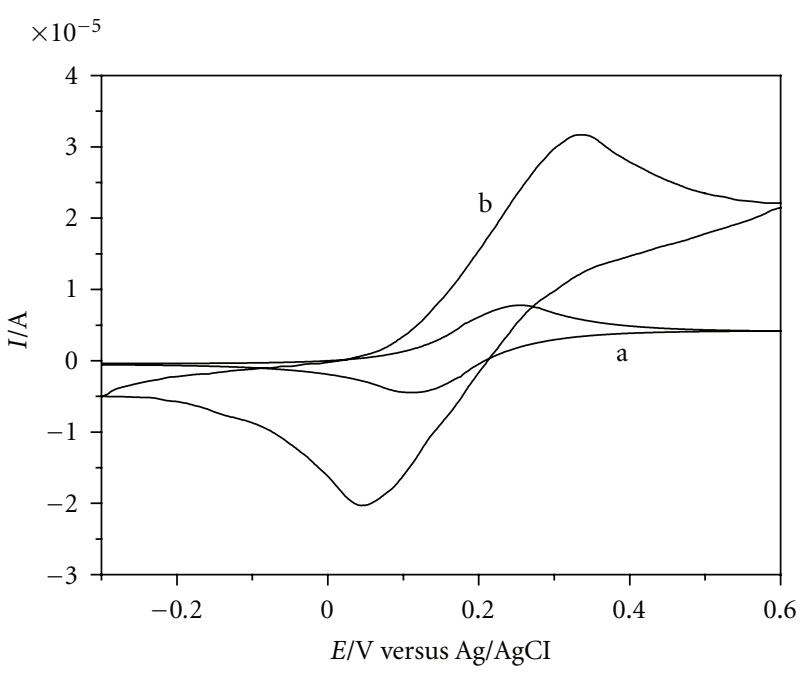

(a) Glassy carbon

(b) Glassy carbon with MWNTs

FIgURE 6: Voltamperometric profiles corresponding to a glassy carbon electrode (GCE): (a) without, and (b) with MWNTs.

as a consequence, hydrogen adsorption is greater. On the basis of these results, we identified this potential value as the electrochemical pretreatment of choice for the activation of the MWNT surfaces. In fact, the voltamperograms presented in Figure 6 were obtained by anodic polarization at this potential value. On the other hand, it is important to point out that the capacity for hydrogen adsorption on MWNTs is, for instance, approximately five times larger using $\mathrm{Ti}$ coverage than that corresponding to bare MWNTs.

\section{Conclusions}

The functionalization of MWNTs and titanium implantation were carried out. Even considering that the attachment of gold particles to nanotubes or, inversely the attachment of nanotubes to gold electrodes, was successfully achieved, the polyelectrolyte may interfere in the titanium fixing procedure. Also, the use of ultramicroelectrodes was unsatisfactory due to the lack of reproducibility in the amount of nanotubes and because the nanotubes length surpassed the diffusion layer. Aside from these results and the corresponding considerations, it was found that the procedure allowed five times higher hydrogen storage and may be susceptible to high improvement, mainly in the amount of atomic titanium bonded, which may cause a significant increase.

In this way, carbon surface functionalization by electrochemical oxidation may provide a great number of functional groups susceptible to nucleophilic attack. The best polarization potential was $750 \mathrm{mV}$. The degree of reactivity of the Ti precursor had a key role in achieving the bonding with nanotubes. The amount of recorded charge was related to the amount of $\mathrm{H}_{2}$ bonded for each potential studied. This was established, since the response of the modified MWNTs changed abruptly with the $\mathrm{H}_{2}$ saturated solution. Anodic pretreatment of the nanostructured surfaces facilitated the 


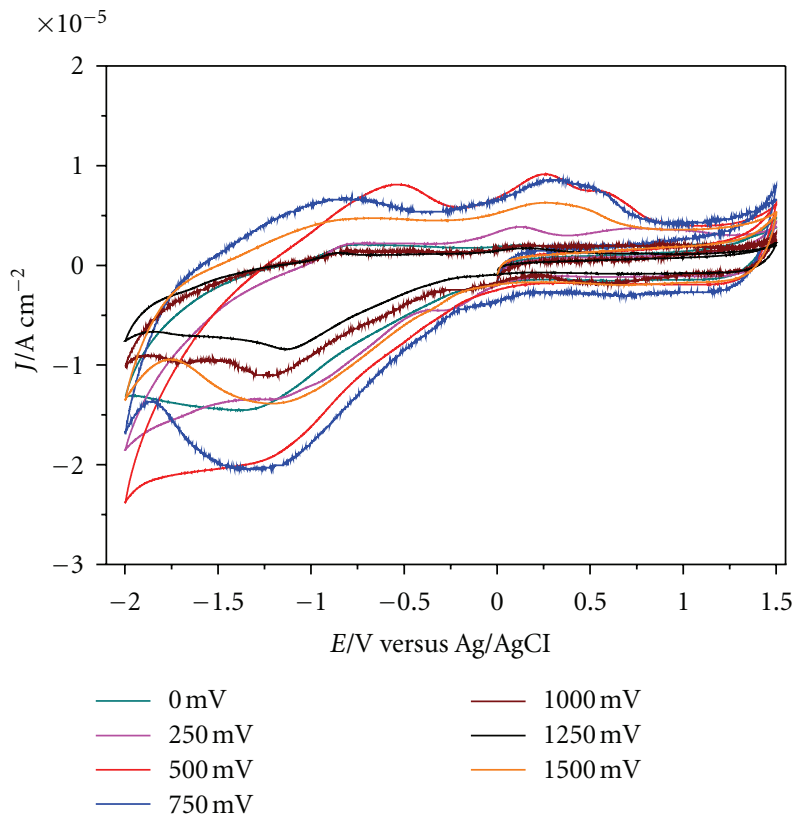

(a)

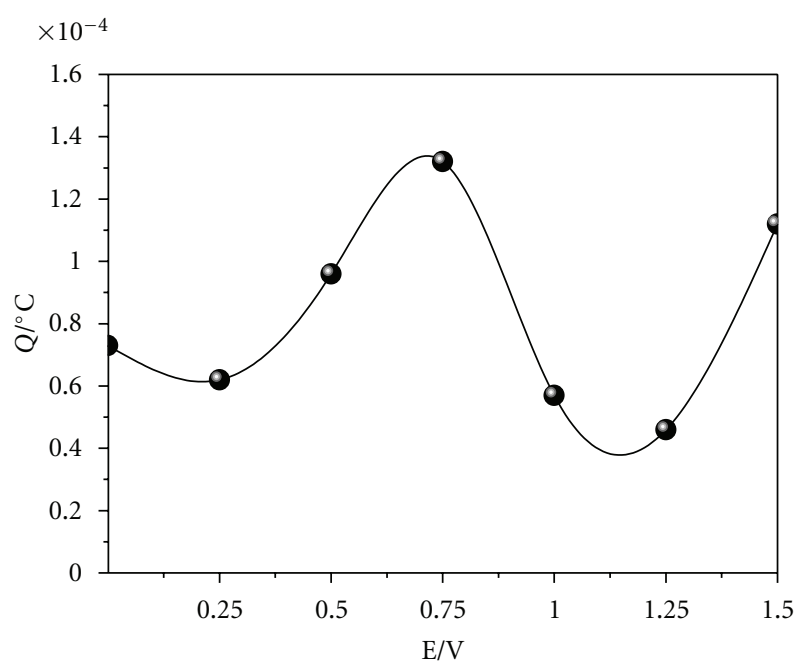

(b)

Figure 7: Profiles obtained by (a) voltamperometry of the nanotubes electrochemically modified with $\mathrm{Ti}$ as a function of the initial polarization, and (b) amount of load corresponding to nanotubes modified with $\mathrm{Ti}$ as a function of the initial polarization.

immobilization of Ti atoms, increasing the hydrogen adsorption of the nanotubes at least five times as compared with nanotubes alone.

\section{Acknowledgments}

The authors gratefully acknowledge the financial support from the Mexican Council for Science and Technology (CONACyT, Grant CB-2009-01 133157). K. M. Brieño Enriquez and J. Ledesma García also acknowledge CONA$\mathrm{CyT}$ for their graduate fellowships. The authors are especially grateful for Darlene Garey of the US Peace Corps for her valuable suggestions to this work.

\section{References}

[1] S. M. Lee and Y. H. Lee, "Hydrogen storage in single-walled carbon nanotubes," Applied Physics Letters, vol. 76, no. 20, pp. 2877-2879, 2000.

[2] F. Lamari Darkrim, P. Malbrunot, and G. P. Tartaglia, "Review of hydrogen storage by adsorption in carbon nanotubes," International Journal of Hydrogen Energy, vol. 27, no. 2, pp. 193-202, 2002.

[3] A. Nikitin, H. Ogasawara, D. Mann et al., "Hydrogenation of single-walled carbon nanotubes," Physical Review Letters, vol. 95, no. 22, Article ID 225507, pp. 1-4, 2005.

[4] A. C. Dillon, K. M. Jones, T. A. Bekkedahl, C. H. Kiang, D. S. Bethune, and M. J. Heben, "Storage of hydrogen in singlewalled carbon nanotubes," Nature, vol. 386, no. 6623, pp. 377379, 1997.

[5] A. C. Dillon, E. Whitney, C. Engtrakul et al., "Novel organometallic fullerene complexes for vehicular hydrogen storage," Physica Status Solidi B, vol. 244, no. 11, pp. 43194322, 2007.

[6] A. C. Dillon and M. J. Heben, "Hydrogen storage using carbon adsorbents: past, present and future," Applied Physics A, vol. 72, no. 2, pp. 133-142, 2001.

[7] P. Santhosh, A. Gopalan, and K. P. Lee, "Gold nanoparticles dispersed polyaniline grafted multiwall carbon nanotubes as newer electrocatalysts: preparation and performances for methanol oxidation," Journal of Catalysis, vol. 238, no. 1, pp. 177-185, 2006.

[8] Y. Feng, T. Yang, W. Zhang, C. Jiang, and K. Jiao, "Enhanced sensitivity for deoxyribonucleic acid electrochemical impedance sensor: Gold nanoparticle/polyaniline nanotube membranes," Analytica Chimica Acta, vol. 616, no. 2, pp. 144-151, 2008.

[9] L. Yuan, M. Yang, F. Qu, G. Shen, and R. Yu, "Seed-mediated growth of platinum nanoparticles on carbon nanotubes for the fabrication of electrochemical biosensors," Electrochimica Acta, vol. 53, no. 10, pp. 3559-3565, 2008.

[10] Y. Umasankar, S. Thiagarajan, and S. M. Chen, "Pinecone shape hydroxypropyl- $\beta$-cyclodextrin on a film of multiwalled carbon nanotubes coated with gold particles for the simultaneous determination of tyrosine, guanine, adenine and thymine," Carbon, vol. 45, no. 14, pp. 2783-2796, 2007.

[11] A. M. Showkat, K. P. Lee, A. I. Gopalan, S. H. Choi, and Y. C. Nho, "Dispersion of gold nanoparticles into thiolfunctionalized carbon nanotubes by $\gamma$-radiation," Diamond and Related Materials, vol. 16, no. 8, pp. 1688-1692, 2007.

[12] X. Kang, Z. Mai, X. Zou, P. Cai, and J. Mo, "A novel glucose biosensor based on immobilization of glucose oxidase in chitosan on a glassy carbon electrode modified with goldplatinum alloy nanoparticles/multiwall carbon nanotubes," Analytical Biochemistry, vol. 369, no. 1, pp. 71-79, 2007.

[13] Q. Kang, L. Yang, and Q. Cai, "An electro-catalytic biosensor fabricated with Pt-Au nanoparticle-decorated titania nanotube array," Bioelectrochemistry, vol. 74, no. 1, pp. 62-65, 2008.

[14] I. Paramasivam, J. M. Macak, and P. Schmuki, "Photocatalytic activity of $\mathrm{TiO}_{2}$ nanotube layers loaded with $\mathrm{Ag}$ and $\mathrm{Au}$ nanoparticles," Electrochemistry Communications, vol. 10, no. 1, pp. 71-75, 2008. 
[15] N. A. Kumar and Y. T. Jeong, "Fabrication of conducting polyaniline-multiwalled carbon nanotube nanocomposites and their use as templates for loading gold nanoparticles," Polymer International, vol. 59, no. 10, pp. 1367-1374, 2010.

[16] X. R. Ye, Y. Lin, C. Wang, M. H. Engelhard, Y. Wang, and C. M. Wai, "Supercritical fluid synthesis and characterization of catalytic metal nanoparticles on carbon nanotubes," Journal of Materials Chemistry, vol. 14, no. 5, pp. 908-913, 2004.

[17] F. Zuliani and E. J. Baerends, "Addition of titanium as a potential catalyst for a high-capacity hydrogen storage medium," Journal of Physics Condensed Matter, vol. 20, no. 6, Article ID 064242, 2008.

[18] X. Qin, X. P. Gao, H. Liu et al., "Electrochemical hydrogen storage of multiwalled carbon nanotubes," Electrochemical and Solid-State Letters, vol. 3, no. 12, pp. 532-535, 2000.

[19] Z. P. Guo, S. H. Ng, J. Z. Wang et al., "Electrochemical hydrogen storage in single-walled carbon nanotube paper," Journal of Nanoscience and Nanotechnology, vol. 6, no. 3, pp. 713-718, 2006.

[20] C. Nutzenadel, A. Zuttel, D. Chartouni, and L. Schlapbach, "Electrochemical storage of hydrogen in nanotube materials," Electrochemical and Solid-State Letters, vol. 2, no. 1, pp. 30-32, 1999.

[21] A. K. M. Fazle Kibria, Y. H. Mo, K. S. Park, and K. S. Nahm, "Electrochemical hydrogen storage behaviors of CVD, AD and LA grown carbon nanotubes in $\mathrm{KOH}$ medium," International Journal of Hydrogen Energy, vol. 26, no. 8, pp. 823-829, 2001.

[22] G. P. Dai, M. Liu, M. Z. Wang, and H. M. Cheng, "Research progress of electrochemical hydrogen storage in carbon nanotubes," New Carbon Materials, vol. 17, no. 3, pp. 70-74, 2002.

[23] P. P. Prosini, A. Pozio, S. Botti, and R. Ciardi, "Electrochemical studies of hydrogen evolution, storage and oxidation on carbon nanotube electrodes," Journal of Power Sources, vol. 118, no. 1-2, pp. 265-269, 2003.

[24] Y. S. Nechaev and O. K. Alekseeva, "Methodological, applied and thermodynamic aspects of hydrogen sorption by graphite and related carbon nanostructures," Uspekhi Khimii, vol. 73, no. 12, pp. 1308-1337, 2004.

[25] Ch. Nützenadel, A. Züttel, Ch. Emmenegger, P. Sudan, and L. Schlapbach, "Electrochemical storage of hydrogen in carbon single wall nanotubes," in Science and Applications of Nanotubes, D. Tománek and R. J. J. Enbody, Eds., Fundamental Materials Research Series, pp. 205-213, Kluwer Academic Publishers, New York, NY, USA, 2002.

[26] C. T. Hsieh, Y. W. Chou, and J. Y. Lin, "Fabrication and electrochemical activity of $\mathrm{Ni}$-attached carbon nanotube electrodes for hydrogen storage in alkali electrolyte," International Journal of Hydrogen Energy, vol. 32, no. 15, pp. 3457-3464, 2007.

[27] M. Li, Y. Li, Z. Zhou, P. Shen, and Z. Chen, "Ca-Coated boron fullerenes and nanotubes as superior hydrogen storage materials," Nano Letters, vol. 9, no. 5, pp. 1944-1948, 2009.

[28] T. Yildirim and S. Ciraci, "Titanium-decorated carbon nanotubes as a potential high-capacity hydrogen storage medium," Physical Review Letters, vol. 94, no. 17, Article ID 175501, 2005.

[29] S. Dag, Y. Ozturk, S. Ciraci, and T. Yildirim, "Adsorption and dissociation of hydrogen molecules on bare and functionalized carbon nanotubes," Physical Review B, vol. 72, no. 15, Article ID 155404, pp. 1-8, 2005.

[30] S. A. Shevlin and Z. X. Guo, "High-capacity roomtemperature hydrogen storage in carbon nanotubes via defectmodulated titanium doping," Journal of Physical Chemistry C, vol. 112, no. 44, pp. 17456-17464, 2008.
[31] S. Bhattacharya, C. Majumder, and G. P. Das, "Hydrogen storage in Ti-decorated $\mathrm{BC}_{4} \mathrm{~N}$ nanotube," Journal of Physical Chemistry C, vol. 112, no. 45, pp. 17487-17491, 2008.

[32] N. Akman, E. Durgun, T. Yildirim, and S. Ciraci, "Hydrogen storage capacity of titanium met-cars," Journal of Physics Condensed Matter, vol. 18, no. 41, article 017, pp. 9509-9517, 2006. 

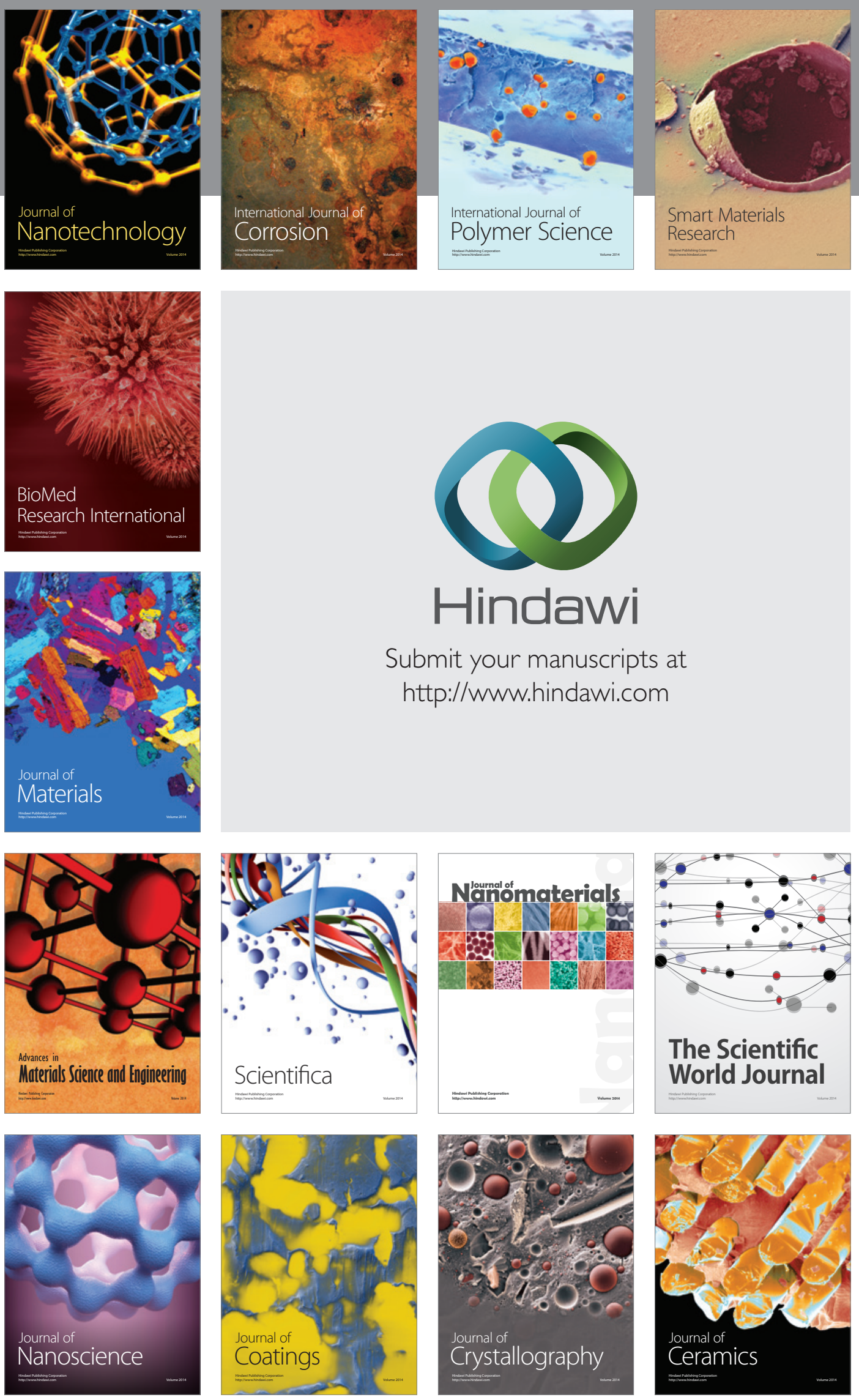

The Scientific World Journal

Submit your manuscripts at

http://www.hindawi.com

\section{World Journal}

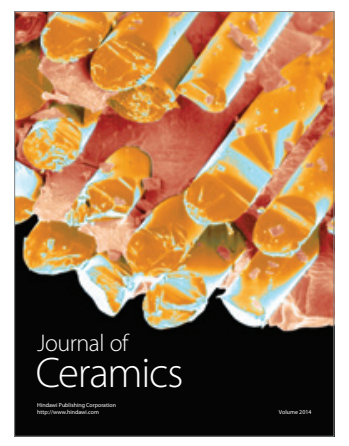

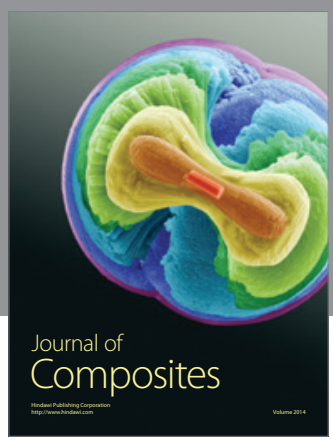
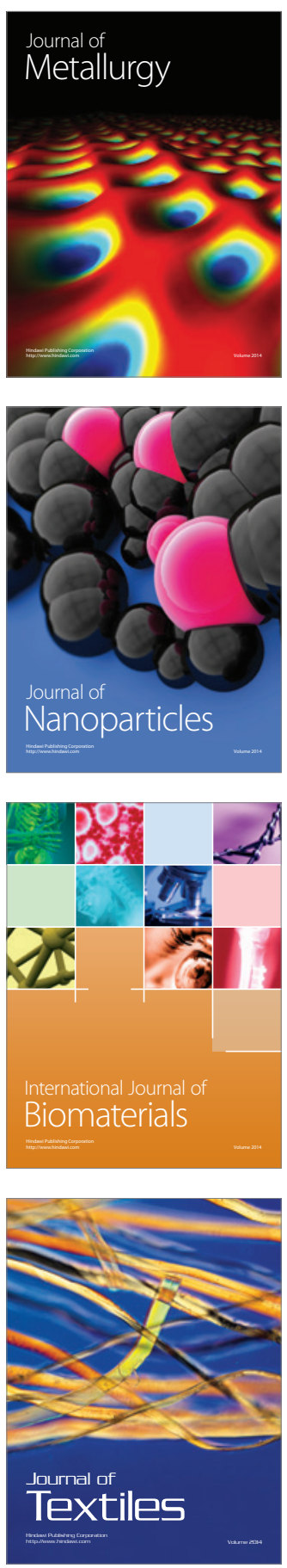